\title{
Laboratory Investigation of Liquid Injectivity in Surfactant-Alternating-Gas Foam Enhanced Oil Recovery
}

\author{
J. Gong ${ }^{1}$ (D) S. Vincent-Bonnieu ${ }^{2}$ - R. Z. Kamarul Bahrim ${ }^{3}$. C. A. N. B. Che Mamat ${ }^{3}$. \\ J. Groenenboom ${ }^{2} \cdot$ R. Farajzadeh ${ }^{1,2} \cdot$ W. R. Rossen ${ }^{1}$
}

Received: 3 April 2018 / Accepted: 30 December 2018 / Published online: 18 February 2019

(c) The Author(s) 2019

\begin{abstract}
Surfactant-alternating-gas (SAG) is a favored method of foam injection, in part because of excellent gas injectivity. However, liquid injectivity is usually very poor in SAG. We report a core-flood study of liquid injectivity under conditions like those near an injection well in SAG application in the field, i.e., after a prolonged period of gas injection following foam. We inject foam [gas (nitrogen) and surfactant solution] into a 17-cm-long Berea core at temperature of $90{ }^{\circ} \mathrm{C}$ with 40 bar back pressure. Pressure differences are measured and supplemented with CT scans to relate water saturation to mobilities. Liquid injectivity directly following foam is very poor. During prolonged gas injection following foam, a collapsed-foam region forms near the inlet and slowly propagates downstream, in which water saturation is reduced. This decline in liquid saturation reflects in part liquid evaporation, also pressure-driven flow and capillary effects on the core scale. In the collapsed-foam region, liquid mobility during subsequent liquid injection is much greater than downstream, and liquid sweeps the entire core cross section rather than a single finger. Mobility in the region of liquid fingering is insensitive to the quality of foam injected before gas and the duration of the period of gas injection. This implies that at the start of liquid injection in a SAG process in the field, there is a small region very near the well, crucial to injectivity, substantially different from that further out, and not described by current foam models. The results can guide the development of a model for liquid injectivity based on radial propagation of the various banks seen in the experiments.
\end{abstract}

Keywords Foam $\cdot$ EOR $\cdot \mathrm{SAG} \cdot$ Injectivity $\cdot \mathrm{CT}$ scan

Electronic supplementary material The online version of this article (https://doi.org/10.1007/s11242-01801231-5) contains supplementary material, which is available to authorized users.

J. Gong

j.gong@tudelft.nl

1 Department of Geoscience and Engineering, Delft University of Technology, 2628 CN Delft, The Netherlands

2 Shell Global Solutions International B.V., 1031 HW Amsterdam, The Netherlands

3 PETRONAS, Kuala Lumpur 50088, Malaysia 


\section{Introduction}

Gas injection is a well-known oil recovery process, either deployed as gas injection alone or in combination with water injection, i.e., in a water-alternating-gas process. However, a gas-injection EOR process can be uneconomical because of poor gas sweep efficiency. The gas injection recovery can be reduced by gravity override or gas breakthrough in highpermeability zones (Lake et al. 2014). This can degrade the gas utilization factor, making the process uneconomical. Foam can be used in enhanced oil recovery to improve gas sweep efficiency (Schramm 1994; Rossen 1996). Foam can be injected into reservoirs mainly in two ways: co-injecting gas and surfactant solution (Stone 1982; Rossen et al. 2010) and injecting gas slugs and surfactant solution slugs, alternatively (Kibodeaux and Rossen 1997). Surfactant-alternating-gas (SAG) is a favored method of foam injection, in part because of its excellent injectivity during gas injection (Matthews 1989; Heller 1994; Shan and Rossen 2004) as well as for operational reasons. Liquid injectivity is the key economical factor for a foam EOR process. However, liquid injectivity is usually considered to be very poor in a SAG process, and the injection well can be fractured during liquid injection (Kuehne et al. 1990; Martinsen and Vassenden 1999).

Most of the literature specifically on liquid injectivity following foam is related to matrix acid diversion (Kibodeaux et al. 1994; Zeilinger et al. 1995; Nguyen et al. 2003; Nguyen et al. 2009). The objective in an acid stimulation process is to maintain low water relative permeability and reduce liquid injectivity to the greatest extent possible. However, in a SAG process, poor liquid injectivity should be avoided, since it slows the injection of liquid slugs and makes a SAG process less economical.

Liquid injectivity directly following foam injection in a foam-acid diversion process was studied in laboratory-scale core-flood experiments. It was found that when liquid was injected following foam, the water relative permeability stayed at nearly the same low value as in foam (e.g., 0.001) (Persoff et al. 1991). Kibodeaux et al. (1994) found that water relative permeability rose by a modest amount, by a factor of about 5 , during liquid injection following foam. The pressure gradient during liquid injection was then constant for a time and then decreased again by a large factor, in a wave-like advance front from the inlet to the outlet of the core. Gas dissolution into unsaturated liquid within liquid fingers penetrating the trapped gas would account for the rise in liquid injectivity.

CT scan experiments of liquid injection directly following foam (Nguyen et al. 2009) confirmed that liquid penetrates foam in one or two fingers in the core and displaces gas within the fingers, leaving foam outside of the fingers trapped in place. Unlike conventional fingering, with an increasingly non-uniform front as it advances, eventually forming fingers, liquid displaces foam only in these fingers directly from the inlet of the core. This suggests that the mechanism is not simply viscous fingering, but trapping of gas in place by the foam. The CT measurements also confirmed that over time liquid saturation rises within the fingers, suggesting that unsaturated liquid dissolves the gas remaining within the fingers over time.

The effect of the phase behavior of gas and liquid during $\mathrm{CO}_{2}$ injection has been investigated (Noh et al. 2007; Pickup et al. 2012). In particular, gas mobility near the well can be affected by water evaporation into injected gas.

However, liquid injectivity in a SAG process, especially after a period of gas injection following foam, remains largely unexplored. In this paper, we report a core-flood study of liquid injectivity under conditions like those near an injection well in SAG application in the field, i.e., after a period of gas injection following foam. 


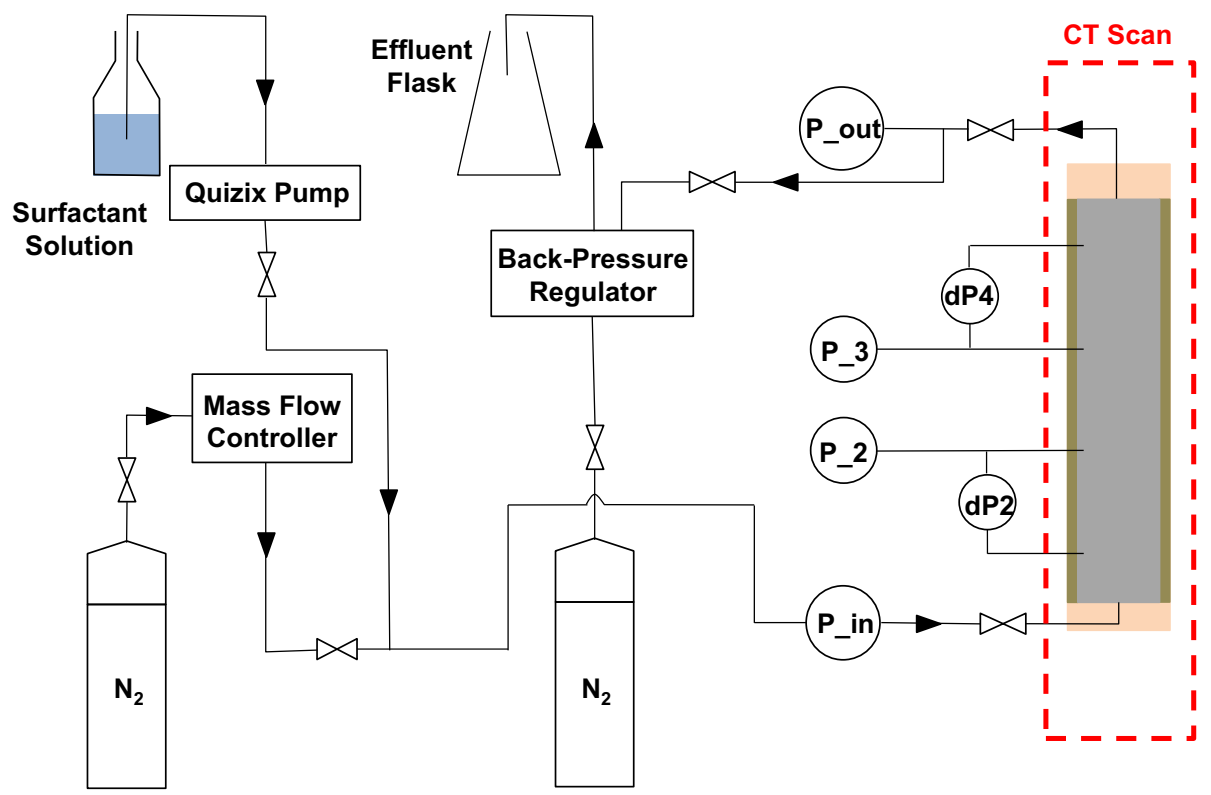

Fig. 1 A schematic of the core-flood experimental setup

We first introduce our experimental setup and procedure and then discuss liquid injectivity under different conditions, especially, the effect of a period of gas injection. Discussion and conclusions are presented at the end.

\section{Experiments}

\subsection{Materials and Experimental Setup}

The core-flood experiments are conducted in a setup schematically shown in Fig. 1. Berea sandstone cores are used in the experiments. The cores are $17 \mathrm{~cm}$ long, with a $4 \mathrm{~cm}$ diameter. The absolute permeability is about $150 \mathrm{mD}$, and porosity is about 0.2 . The core is glued and fitted into a PEEK (polyether ether ketone, a semi-crystalline thermoplastic) core holder, which is suitable for CT scan analysis due to its low X-ray attenuation. Liquid and gas are injected from the bottom of the core holder. The injection velocities of gas and liquid are controlled by a mass flow controller and a Quizix pump, separately. Two differential pressure transducers and four absolute pressure transducers are applied to measure the pressure drops in five sections along the core, and the absolute pressures at positions starting from the inlet to the outlet of the core. The core holder is placed vertically in an oven to maintain a constant temperature $\left(90^{\circ} \mathrm{C}\right)$. Moreover, at the pressure gradients of most of our data, gravity effects would not be expected to be significant. The outlet of the core holder is connected to a backpressure regulator and a gas cylinder to maintain a constant back pressure (40 bar). A confining pressure around the core, equal to the injection pressure, is applied. In a conventional Hassler core holder, the confining pressure must greatly exceed the core pressure in order to press the rubber sleeve against the outer surface of the core. Our core is first coated with a layer of epoxy that penetrates a short distance into the core. The purpose of the confining pressure is 
simply to prevent the inner pressure from greatly exceeding the outer pressure and causing the epoxy coating to crack. By having a confining pressure equal to the largest pressure in the core (that at the inlet), we achieve this purpose.

The surfactant used in the experiments is alpha olefin sulfonate (Witconate AOS, produced by AkzoNobel), due to its good stability at high temperature. The surfactant solution is at a concentration of $0.5 \mathrm{wt} \%$. The brine we use is synthetic seawater with five salts (sodium chloride, sodium sulphate, magnesium chloride, calcium chloride, and potassium chloride). The brine is at $3 \mathrm{wt} \%$ salinity. Nitrogen is supplied from a 220-bar gas cylinder. The same rock is used for all the experiments presented in this work, except the CT scan experiments. A single, similar core is used in the CT scan experiments.

A medical CT scanner (Siemens Somatom) is used to monitor the water saturations and relate the water saturations to the mobilities in some experiments. In the CT scan experiments, the oven is replaced by a thermal jacket, with silicon oil flowing inside, to maintain the core temperature at $90{ }^{\circ} \mathrm{C}$. In the CT experiments, the core is held vertical. The entire core is scanned at one time, which allows us to obtain the water saturation profiles along the core and at cross sections. The resolution of each image is $521 \times 521$ pixels for both the crosssectional and axial profiles. The slice thickness is $3 \mathrm{~mm}$.

\subsection{Procedure}

We inject nitrogen foam, gas (nitrogen), and surfactant solution into a 17-cm-long Berea core at temperature of $90{ }^{\circ} \mathrm{C}$ with a 40 bar back pressure. Pressure differences are measured separately across five sections of the core and supplemented with CT scans to relate water saturation to mobilities. The two sections close to the inlet and outlet, respectively, are $2.2 \mathrm{~cm}$ long, and the three middle sections are each $4.2 \mathrm{~cm}$ long. In order to minimize the entry region and capillary end effects, the inlet and outlet sections are not considered in our analysis.

The experiments aim to examine liquid injectivity in different situations. First, we examine liquid injectivity directly following foam. We then examine liquid injectivity following different periods of gas injection after foam, to reflect liquid injectivity near the well in a SAG process. In all experiments, foam is generated initially by co-injecting gas and surfactant solution. SAG field application does not begin with co-injection of surfactant solution and gas. We are concerned, though, that a short core may not allow foam to achieve local equilibrium in the first pore volume injection of dynamic SAG core flood. Therefore, we start with foam in the core and follow it with gas.

\section{Results}

\subsection{Foam Quality Scan}

In order to identify the high-quality and low-quality foam regimes (Alvarez et al. 2001; Boeije and Rossen 2015) for the Berea core used in this study, we first conduct a foam quality (gas fractional flow) scan at a single superficial velocity $2 \mathrm{ft} /$ day (Fig. 2). As shown in Fig. 2, the transition foam quality between the regimes is about 0.8 . Thus, here we conduct subsequent experiments with a gas fraction 0.95 and 0.6 , to represent initial foam in the high-quality and low-quality regimes, separately. We present a model fit to the foam quality scan in a separate paper (Gong et al. 2018). The transition foam quality from the model fit is around 0.8 . 
Fig. 2 Scan of apparent viscosity versus foam quality (gas fractional flow)

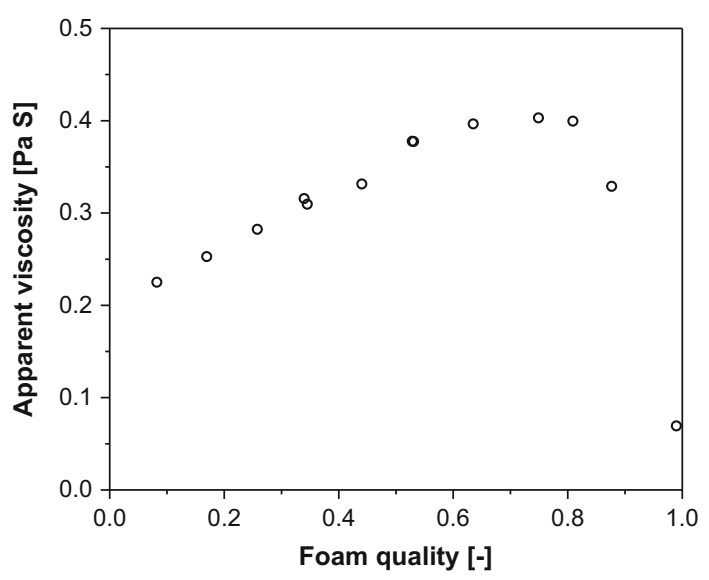

(a)

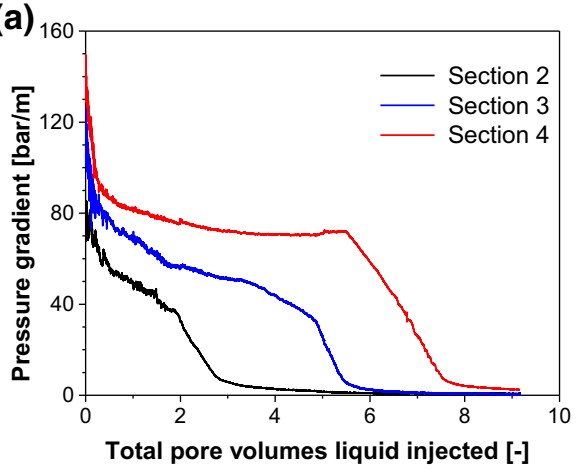

(b)

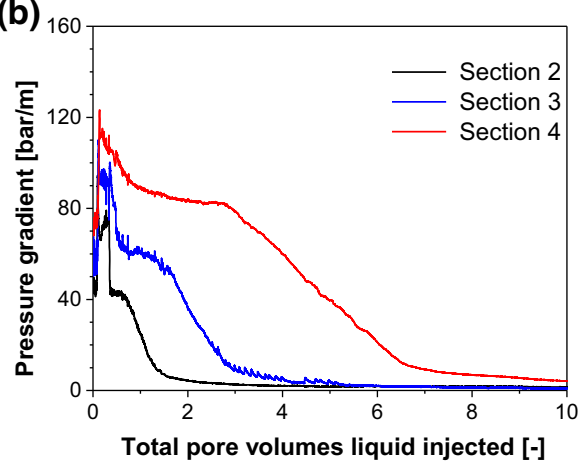

Fig. 3 Pressure gradient during liquid injection directly following foam: a 0.6-quality (low-quality regime) foam, b 0.95 -quality (high-quality regime) foam

\subsection{Liquid Injection Directly Following Foam}

In this section, we present results for the case that liquid injection directly follows steadystate foam. As shown in Fig. 3, in the very beginning, liquid enters the core with low relative permeability (the initial rise in pressure gradient). Liquid then penetrates foam in a few fingers; within those fingers, liquid displaces gas with low mobility. This happens very quickly, since not much gas is displaced; therefore, the fingers advance quickly. Overall, the pressure gradient during this period is still large (the plateau in pressure gradient in Fig. 3). Thereafter, virtually all liquid travels through the fingers; gas outside of fingers stays trapped in place. During this period, mobility holds constant, as liquid fingers through without dissolving gas. Mobility then rises subsequently, reflecting displacement or dissolution of gas trapped within the fingers into unsaturated liquid (the second decline in pressure gradient). This happens progressively in a front that propagates from the inlet to the outlet of the core. Gas surrounding the fingers remains trapped. Over time, the fingers also grow very slowly outwards as they dissolve surrounding trapped gas (see Nguyen et al. 2009).

Figure 3 shows the comparison of the pressure gradient during liquid injection at the same velocity, $2 \mathrm{ft} /$ day, following different qualities of foam (0.6 and 0.95). In the beginning, the maximum pressure gradients for all the three sections during liquid injection following 


\section{(a)}
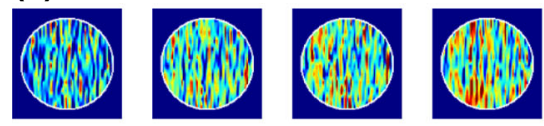

(b)
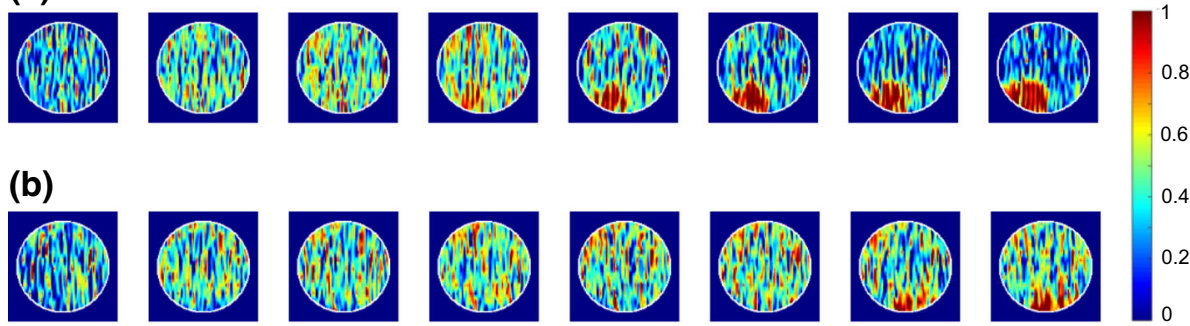

0.4

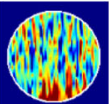

0.2

Fig. 4 Reconstructed cross-sectional water saturation profile at two positions and various times. a $4.4 \mathrm{~cm}$ from the inlet (in Sect. 2); b $13 \mathrm{~cm}$ from the inlet (in Sect. 4). Columns correspond to: steady-state 0.95-quality foam, injection of 0.9 PV, 1.5 PV, 3.2 PV, 5.7 PV, 8.1 PV, 12.7 PV, and 16.9 PV liquid injected following steady-state foam, respectively

0.95-quality foam are lower than that for 0.6-quality foam. Thereafter, the plateau values in pressure gradients are relatively insensitive to the quality of foam injected before liquid injection. The plateau tends to last longer for lower-quality foam. For Sect. 4, for example, the plateau in pressure gradient during liquid injection following 0.6-quality foam lasts about 5 PV, while it lasts only 2 PV for liquid injection following 0.95-quality foam. However, while there is a substantial difference in the pore volumes liquid injected to displace or dissolve the gas in the first three sections, that difference has largely disappeared in Sect. 4 (7.5 PV liquid injection after 0.6-quality foam, and 6.5 PV liquid injection after 0.95-quality foam, separately).

Our pressure gradients are indeed much greater than those would occur from an injection well. They are not so far above those occurring near an injection well, however, which is the region most important to injectivity. So we believe our experimental results are relevant, at least qualitatively, to the behavior in the near-well region.

The CT scan results mostly confirm the mechanisms that determine the behavior discussed above. For CT scan experiments, we use a different Berea core, with similar permeability and porosity as the core used for the experiments shown above.

As presented in Fig. 4a, at a position $4.4 \mathrm{~cm}$ from inlet, a liquid finger is visible after about 3.2 PV surfactant solution injection (image in column 4). As more liquid is injected, the finger becomes more "red," which indicates gas within the finger has dissolved into liquid or been displaced. The liquid finger also becomes wider over time (images in columns 4-7). The region outside of the fingers becomes more "blue" (water saturation decreases), which implies gas outside of the liquid finger expands as pressure declines, but stays trapped.

At a position $13 \mathrm{~cm}$ from inlet, the liquid finger is visible after 12.7 PV surfactant solution injection (image in column 7 in Fig. 4b). The liquid finger is about as well-developed as it is closer $(4.4 \mathrm{~cm}$ from the inlet) to the core inlet. The liquid finger forms, propagates downstream, and develops roughly in the same way for comparable pore volumes of liquid injection scaled by the cumulative pore volume from a given position back to the inlet. Below we formalize this scaling as "local pore volumes."

Figure 5 shows the average water saturation at positions in Sects. 2 and 4 inside (Fig. 5a) and outside (Fig. 5b) of the liquid finger. As shown in Fig. 5a, water saturation within the finger changes little during the plateau in pressure gradient (which lasts until about $1 \mathrm{PV}$ liquid injection for Sect. 2, and 7 PV liquid injection for Sect. 4); it increases during the second decline in pressure gradient as gas within the finger is dissolved or displaced. At the same time, water saturation falls outside of the finger in Sect. 2; one possible reason is gas 

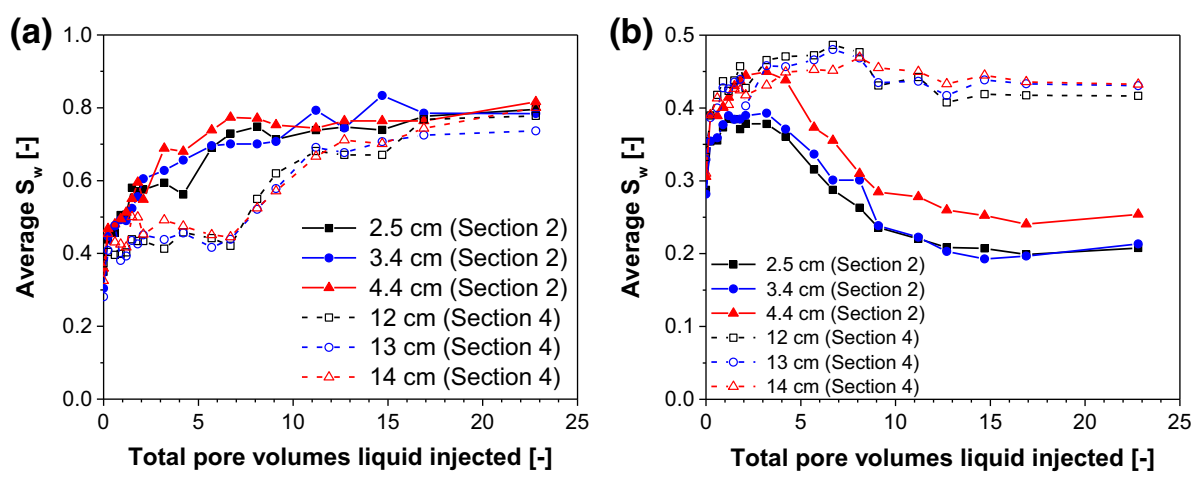

Fig. 5 Average water saturation at different distances from inlet during liquid injection following 0.95-quality foam. a Within the liquid finger; $\mathbf{b}$ outside of the liquid finger

expansion as pressure falls, driving down water saturation there. No major changes in water saturation outside the finger are evident in Sect. 4.

\subsection{Liquid Injection Following a Period of Gas Injection After Foam}

In this series of experiments, we examine the liquid injectivity following various periods of gas injection. The "local pore volume" (LPV) is defined here as the volume injected divided by the cumulative pore volume from a given position back to the inlet. The "total pore volume" $(\mathrm{PV})$ is based on the pore volume of the core.

During gas injection, as shown in Fig. 6, pressure gradients in all three sections first decline to a plateau and hold there for a time, followed by a second decline. The second decline in pressure gradient propagates in a wave-like manner from the inlet toward the outlet. Mobility changes little until a large volume of gas is injected. The second decline in pressure gradient finishes after about 120 PV gas injection for Sect. 2 (316 LPV), 180 PV for Sect. 3 (290 LPV), and 360 PV for Sect. 4 (400 LPV). On average, after about 340 LPV gas injection, mobility increases greatly, which indicates that foam completely collapses or greatly weakens within this region (Fig. 6). Thus, during a period of gas injection, gas at first doesn't destroy foam, though it weakens foam (i.e., the plateau in pressure gradient). Foam then collapses or is greatly weakened (the second decline in pressure gradient), when a sufficient volume of gas has flowed through. For illustration, we call the distance from the inlet at which the apparent major weakening or collapse of foam has happened $x_{c}$ (Fig. 7). Position $x_{c}(t)$ advances with a dimensionless velocity approximately (1/340). Beyond this region, foam weakens, but does not collapse (the plateau in pressure gradient). This dimensionless velocity may partly depend on the gas injection rate, since different flow rates would correspond to different pressure gradients, which is one of the mechanisms displacing water and drying out the foam. Capillary pressure gradients and water evaporation into gas are likely to be involved as well. In a field application, if enough gas was injected to fill the pore volume out to a radius of $100 \mathrm{~m}$, then the region within a radius of $1 \mathrm{~m}$ would have experienced 10,000 pore volumes gas injection. We are primarily interested in the near-well region because of its importance to injectivity.

CT scans are conducted to monitor the changes of water saturation. Due to the time limitation of our CT scanner and large volumes of gas injection needed, we use a larger gas injection velocity, about $60 \mathrm{ft} / \mathrm{day}$, for the CT scan experiments. Increasing gas injection 
Fig. 6 Pressure gradient during gas injection following 0.95-quality (high-quality regime) foam. The superficial gas injection velocity is about $6 \mathrm{ft} /$ day

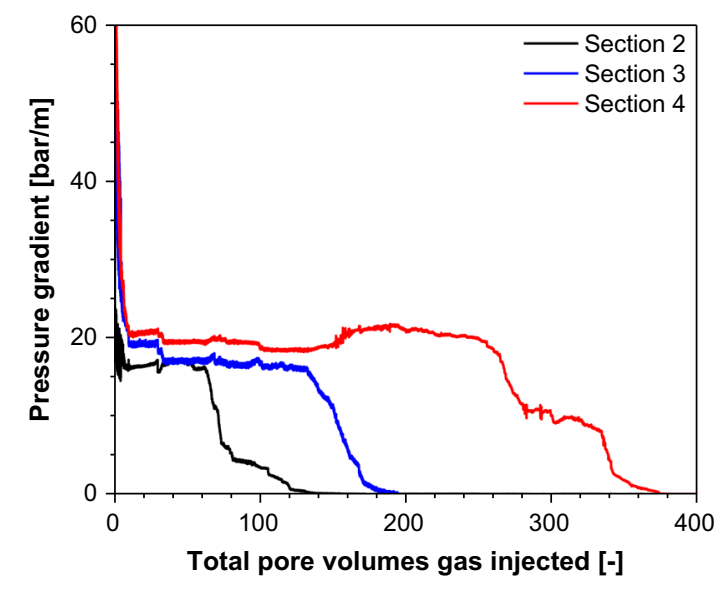

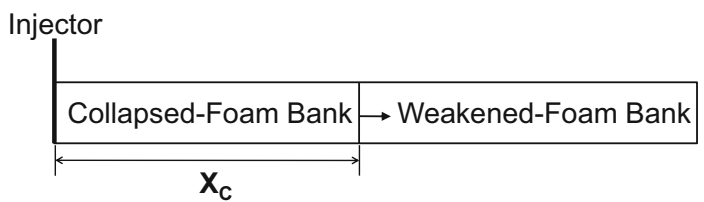

Fig. 7 Banks during gas injection period in SAG process

velocity speeds up the decline in pressure gradient. In the CT scan experiments, with the larger injection velocity, apparent major weakening or collapse of foam happened after about 150 LPV gas injection. Capillary effects are significant on the core scale as well. Using different cores might also lead to different results. But, whatever the driver is, in both of the cases, the collapse or abrupt weakening of foam moves as a front from the inlet to the outlet of the core as more gas is injected.

Figure 8 presents the water saturation profile along the axis of the core during gas injection from CT measurements. The middle part of the images is noisy, so we do not consider this part in our analysis. Foam dries out near the inlet, corresponding roughly to the period of decline in pressure gradient. The drying front propagates from the inlet toward the outlet (yellow arrow in Fig. 8). Water saturation also decreases at other positions along the core (red oval in Fig. 8), but not as much as at the inlet. This is confirmed by the cross-sectional average water saturation shown in Fig. 9. Pressure decline can lead to expansion of trapped gas that either drives water flow downstream or allows liquid to evaporate into gas; both mechanisms drive down water saturation. Foam weakening occurs as the combined result of pressuregradient-driven flow, evaporation of water, and capillary effects. Scaling up therefore can only be approximate. In our experiments, the major weakening or collapse of foam happens at a water saturation $S_{\mathrm{w}}$ of about 0.2 (Fig. 9). The decline in $S_{\mathrm{w}}$ is more abrupt in Sect. 4 than Sect. 2. It is possible that this reflects sharpening of fronts as they move downstream, a greater distance from the entrance effects, or lower pressure (and more rapid water evaporation into the larger gas volume) downstream.

As discussed above, water evaporation into flowing gas is expected to be an important reason for foam weakening or collapse during the gas injection period. Figure 10 shows the comparison of the water saturation averaged over cross sections at various positions, obtained from the CT measurements, and an estimate of the average water saturation from that position back to the inlet based on the amount of water that would evaporate into the 

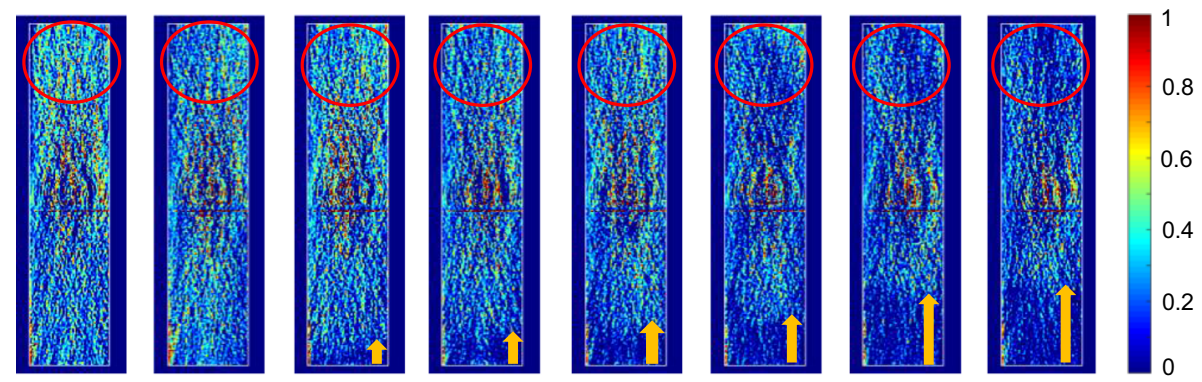

Fig. 8 Water saturation profile along the axis of the core during gas injection at $60 \mathrm{ft} /$ day superficial velocity following 0.95 -quality foam. Image 1: steady-state 0.95 -quality foam; images 2 to 8 : advance of foam collapse front as more gas is injected
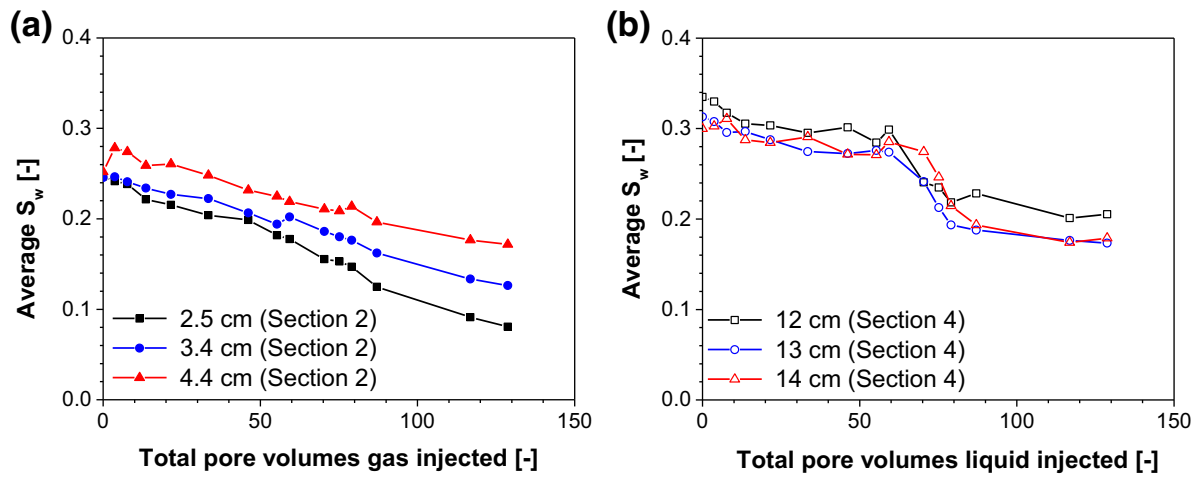

Fig. 9 Average water saturation of cross sections at various positions in a Sect. 2 and $\mathbf{b}$ Sect. 4

given volume of injected gas. The details of the calculation of the evaporation effect are presented in "Appendix." It includes several simplifying assumptions and ignores the effects of capillary pressure drawing water upstream to replace water that evaporated. We present it simply to indicate that the change in water saturation expected from evaporation is significant on the core scale of the saturation changes seen in our experiments. Evaporation would be expected to play a major part in the drying out and collapse of foam during a long period of gas injection.

The previous period of gas injection has a major impact on the subsequent liquid injectivity. As discussed above, two regions are observed after a period of gas injection: a region of collapsed foam near the inlet $\left(x<x_{c}\right)$ and a region of weakened foam further away $\left(x>x_{c}\right)$.

For $x<x_{c}$ (the region of collapsed foam), during the following liquid injection, liquid sweeps whole cross sections. Some gas is still trapped behind the liquid front, but liquid is not fingering in this collapsed-foam region (Fig. 11a). Foam in the whole core collapses after a sufficient amount of gas is injected.

Figure 12 shows the comparison of the sectional pressure gradients during liquid injection following different periods of gas injection. After about $135 \mathrm{PV}$ gas injection, the collapsedfoam region penetrates into Sect. 2 (Fig. 12a). It fills Sects. 2 and 3 after 245 PV gas injection (Fig. 12b). The pressure gradient in Sect. 3 during liquid injection following $135 \mathrm{PV}$ gas is approximately $50 \mathrm{bar} / \mathrm{m}$, which is comparable to the pressure gradient of Sect. 3 during liquid injection directly following foam (Fig. 3b). It is only about $5 \mathrm{bar} / \mathrm{m}$ during liquid injection 
Fig. 10 Comparison of average water saturation calculated based on CT measures (CT) and effects of liquid evaporation (EVA), discussed in "Appendix", at various positions in Sect. 2 of the core

(a)
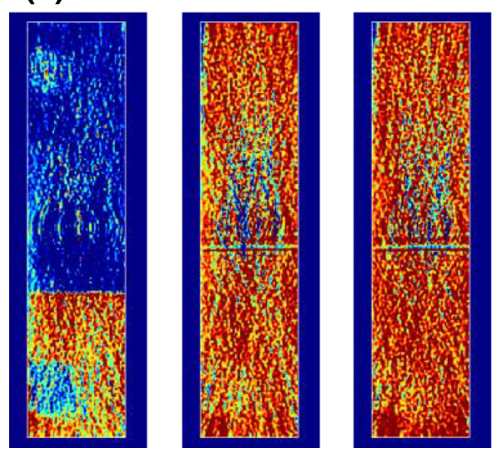

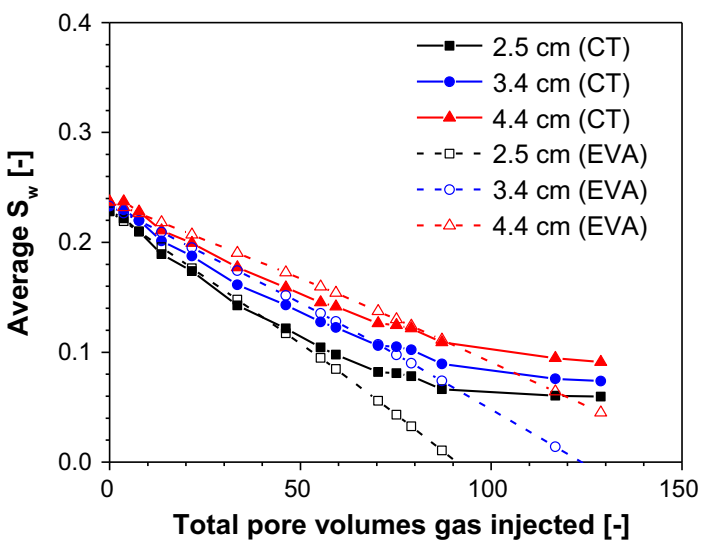

(b)
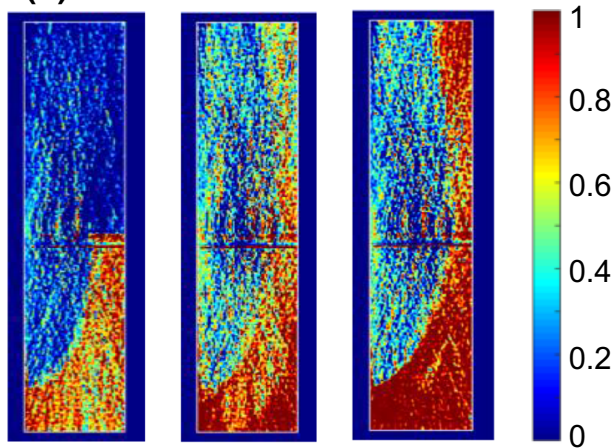

Fig. 11 Water saturation profile along the axis of the core during liquid injection after a period of gas injection. a The collapsed-foam region fills the entire core. b There is a collapsed-foam region near the inlet and a weakened foam region beyond it. From left to right in each sequence, more liquid is injected
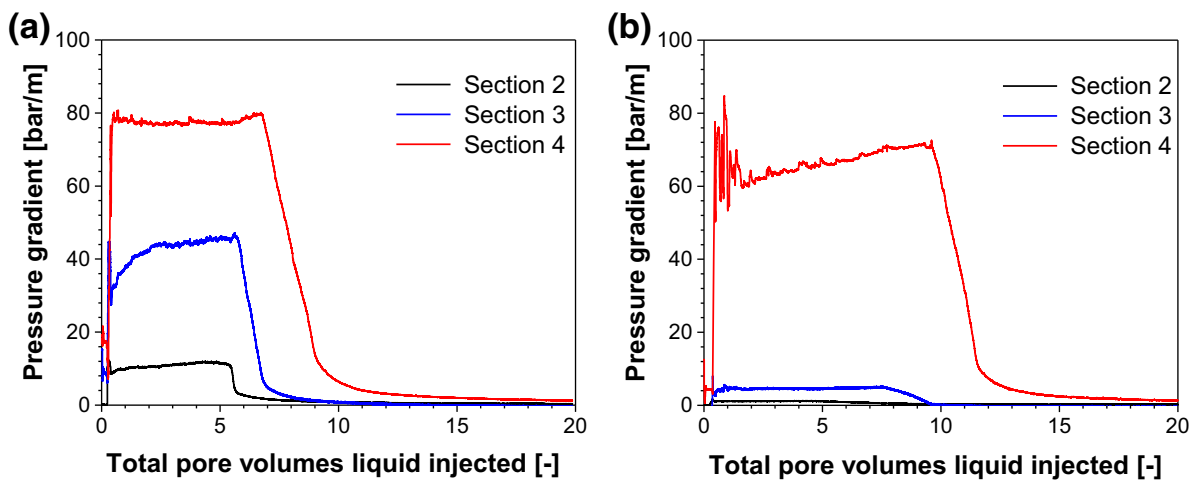

Fig. 12 Pressure gradient during liquid injection after a period of gas injection: a $135 \mathrm{PV}$ gas injection and b $245 \mathrm{PV}$ gas injection

after 245 PV gas was injected. Similarly, the pressure gradients in Sect. 4 during liquid injection following $135 \mathrm{PV}$ gas injection (Fig. 12a) and directly following foam (Figure 3b) 
(a)

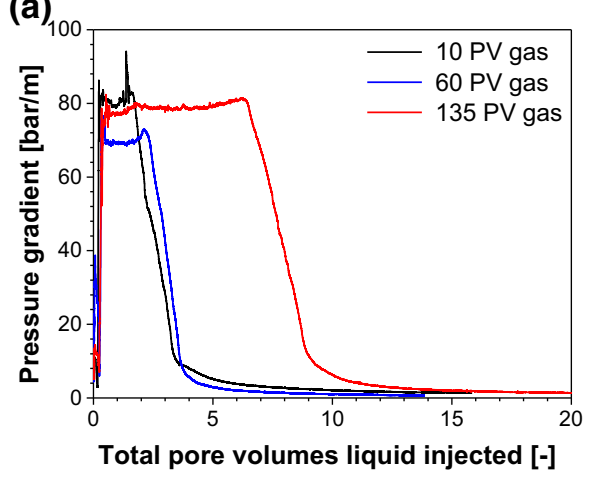

(b)

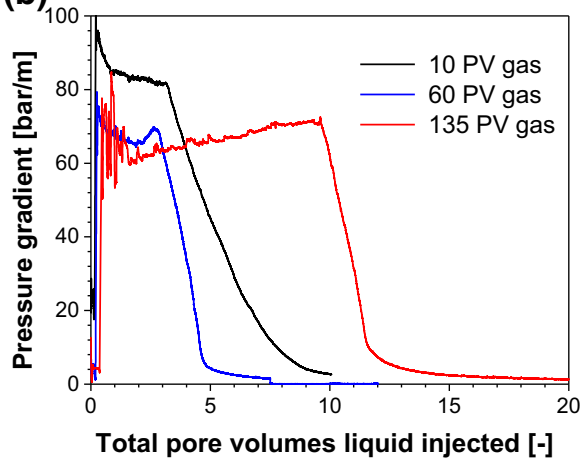

Fig. 13 Pressure gradient in Sect. 4 during liquid injection following different periods of gas injection after injection of foam of different qualities. a 0.6-quality foam (low-quality regime) before gas injection; b 0.95 quality foam (high-quality regime) before gas injection. Foam in Sect. 4 is not collapsed or greatly weakened in these cases

are both about $80 \mathrm{bar} / \mathrm{m}$, which implies that the foam strength in these two cases is nearly the same; foam does not collapse nor greatly weakened in Sect. 4 after 135 PV gas injection. However, the pressure gradient during liquid injection after $245 \mathrm{PV}$ gas injection is lower (about $70 \mathrm{bar} / \mathrm{m}$ ), since the collapsed-foam region partly penetrates into Sect. 4.

Evidently, for the liquid injection period, the pressure gradient or liquid injectivity is strongly affected by the size of the gas slug previously injected. In the collapsed-foam region after a prolonged period of gas injection, mobility is relatively high, which indicates that a long period of gas injection breaks foam, making subsequent liquid injection easier.

For $x>x_{c}$ (region of weakened foam), liquid fingers through foam as more liquid is injected. As shown in Fig. 11b, liquid first flows through the whole cross sections near the inlet $\left(x<x_{c}\right)$, then through part of the cross sections $\left(x>x_{c}\right)$. Foam around the finger stays trapped. As shown in Fig. 12a, the pressure gradient in Sect. 4 during liquid injection following $135 \mathrm{PV}$ gas is approximately $80 \mathrm{bar} / \mathrm{m}$, which is comparable to the case of liquid injection directly following foam (Fig. 3b). Clearly, during liquid injection, the pressure gradient in the weakened foam region beyond the collapsed-foam region is not significantly affected by the previous period of gas injection. In this region, mobility is relatively low, similar to that when liquid is injected directly following foam. Liquid injection after a relative "short" period of gas injection encounters a region of plentiful trapped gas, since foam is not collapsed or greatly weakened.

As shown in Fig. 13, the pressure gradient in the region of weakened foam $\left(x>x_{c}\right)$ during liquid injection (the plateau value) is relatively insensitive to the gas-slug size before liquid injection. The plateau tends to be longer for a larger gas slug injected previously.

Figure 14 compares the pressure gradient during liquid injection following a same amount of gas injection (60 PV) after foam with different qualities (0.6 and 0.95). Foam in Sect. 3 (Fig. 14a) and Sect. 4 (Fig. 14b) is not collapsed or greatly weakened. Apparently, the plateau in pressure gradient is not greatly affected by the foam quality before gas injection.

To summarize, during liquid injection following a period of gas injection, liquid first fills the collapsed-foam region and then fingers through the weakened foam region beyond of it. Subsequently, gas within the liquid finger dissolves into unsaturated liquid or is displaced and mobility rises substantially (Fig. 15).

We also conducted experiments with brine, with no surfactant, injected directly following foam and following a period of gas injection after foam (results can be found in supplementary 
(a)

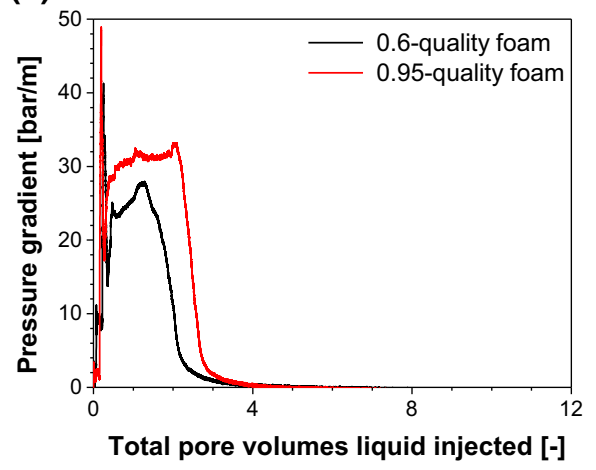

(b)

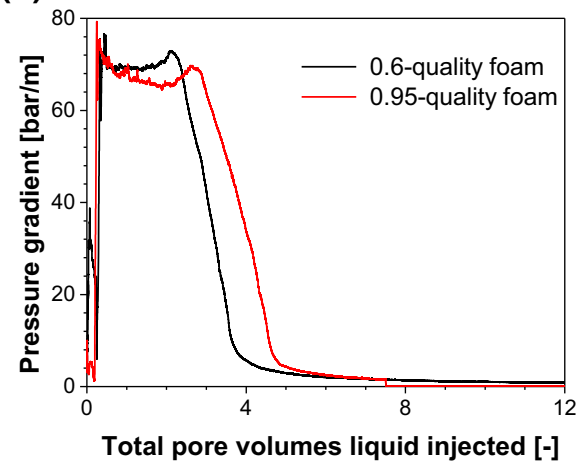

Fig. 14 Pressure gradient in different sections of the core during liquid injection following $60 \mathrm{PV}$ gas injection after 0.6-quality foam (low-quality regime) and 0.95-quality foam (high-quality regime). a Section 3 and b Sect. 4. Foam is not collapsed or greatly weakened in the two sections in these cases

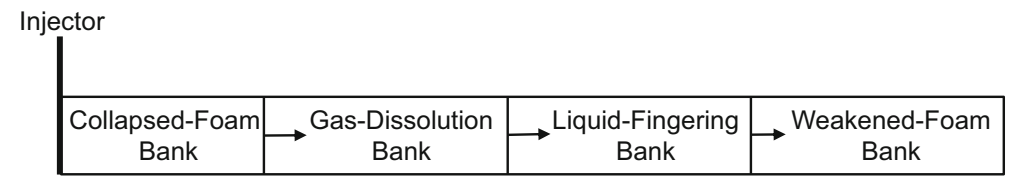

Fig. 15 Banks during liquid injection period in SAG process

material to the paper). We found little difference from the experiments injecting surfactant solution, as did Nguyen et al. (2009).

\section{Conclusions and Discussion}

Liquid injectivity directly following foam is very poor, as shown in previous studies. Liquid first fingers through the trapped foam. It then dissolves or displaces gas trapped within the fingers, and overall mobility rises sharply.

During prolonged gas injection following foam, two regions are observed: the collapsedfoam region and the weakened-foam region. A region forms near the inlet and slowly propagates downstream in which gas mobility is much greater; this indicates that foam collapses or greatly weakens in this region. The abrupt rise in mobility appears to reflect the decline in water saturation below about 0.2 in our experiments. This decline in liquid saturation reflects in part liquid evaporation and also pressure-driven flow and capillary effects on the core scale.

During the subsequent liquid injection period, liquid first quickly fills the collapsed-foam region and then fingers through the weakened-foam region beyond of it. Subsequently, a second region develops within the liquid-fingering region, in which gas within the fingers dissolves into unsaturated liquid or is displaced and mobility rises substantially. There is a rough correspondence between the amount of gas expected to dissolve into unsaturated liquid and the change in water saturation within the finger in Fig. 5a. About $0.012 \mathrm{PV}$ of gas would dissolve into $1 \mathrm{PV}$ of brine at $90{ }^{\circ} \mathrm{C}$ (O'Sullivan and Smith 1970). The process is complicated by pressure being non-uniform along the core and changing with time. However, if brine flows 
through a fraction of the core cross section (i.e., 1/9), $4 \mathrm{PV}$ of liquid injection would dissolve enough gas to change water saturation within the finger by $[0.012 \times 4 \times 9] \approx 40 \%$, which is in agreement with the change in $S_{\mathrm{w}}$ as shown in Fig. 5a. Given the low mobility of gas expected at the high water saturation within the finger, and low mobility of liquid outside the finger, we believe that gas dissolution is the dominant factor in the increasing mobility within the finger over time.

The mobility of injected liquid is much greater in the collapsed-foam region than downstream, and liquid sweeps the entire core cross section rather than a single finger. Beyond the collapsed-foam region, liquid fingers through trapped foam just as in liquid injection directly following foam. Mobility in the weakened-foam region is insensitive to the quality of foam injected before gas and the duration of the period of gas injection (though the size of weakened-foam region depends on the period of gas injection).

These results suggest that the period of gas injection has a major impact on the subsequent liquid injectivity. There is a small region formed during gas injection very near the well, crucial to overall injectivity, in which liquid mobility is much greater than that further out. From the experimental data, we can estimate the dimensionless velocities and mobilities of the various banks that determine liquid injectivity.

The results can guide development of a model for liquid injectivity based on radial propagation of the various banks observed in the experiments (Gong et al. 2018). We find that conditions very near the well, crucial to injectivity, are substantially different from those further out, and are not described by current foam models. However, the effects of pressuregradient-driven flow, liquid evaporation and capillary effects which are responsible for foam collapse during gas injection may not scale up simply to the field, and to other temperatures and pressures. Liquid fingering, which is important to liquid injectivity, is also difficult to extrapolate from core scale to radial flow around a well.

Our experimental results reflect the effects of temperature, pressure, surfactant formulation, gas, porous medium, etc. In particular, the evaporation of water during gas injection and dissolution of gas during liquid injection both depend on vapor pressure and the solubilities of gas and liquid. Moreover, the water saturation for foam collapse would be sensitive to all these factors. For each new field application, a new set of experiments should be conducted, and a new set of parameters fit to those results would be needed.

In the field, some oil would be left behind. Oil will certainly complicate the issues discussed in this work. We have done experiments with residual oil (not reported in this paper). Oil makes foam weaker, but the overall behavior during the gas and liquid injection periods is similar to the cases without oil. Our continuing work aims to examine this effect further.

Acknowledgements We gratefully thank PETRONAS and Shell Global Solution International B.V. for supporting and allowing us to publish this work. We also thank the technical support of Michiel Slob and Ellen Meijvogel-de Koning at the Laboratory Geoscience and Engineering of Delft University of Technology.

Open Access This article is distributed under the terms of the Creative Commons Attribution 4.0 International License (http://creativecommons.org/licenses/by/4.0/), which permits unrestricted use, distribution, and reproduction in any medium, provided you give appropriate credit to the original author(s) and the source, provide a link to the Creative Commons license, and indicate if changes were made.

\section{Appendix}

The amount of water that evaporates into dry gas can be calculated from the equation of state:

$$
P V=n R T
$$


Fig. 16 Schematic of liquid evaporation into dry gas

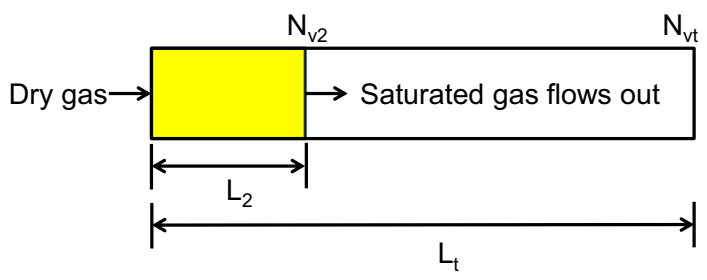

where $n$ is the number of moles of water in the vapor, $P$ is the vapor pressure of water $\left(7 \times 10^{4} \mathrm{~Pa}\right), V$ is the volume of flowing dry gas, $R$ is the universal gas constant $\left(8.314 \mathrm{~m}^{3}\right.$ $\left.\mathrm{Pa} \mathrm{K}^{-1} \mathrm{~mol}^{-1}\right), T$ is the temperature $\left(363.15 \mathrm{~K}\right.$, i.e., $\left.90{ }^{\circ} \mathrm{C}\right)$ in this study. According to the calculation, $418 \mathrm{~cm}^{3}$ liquid water evaporates into $1 \mathrm{~m}^{3}$ gas. In other words, $1 \mathrm{PV}$ dry gas evaporates $4.18 \times 10^{-4}$ PV liquid.

The pore volumes of gas flowing past a given position ("local pore volumes") can be calculated as follows:

$$
N_{v 2}=N_{v t}\left(\frac{L_{t}}{L_{2}}\right)
$$

where $N_{v 2}$ is the local pore volumes of gas passing the given position, $N_{v t}$ is the pore volumes of gas based on the entire pore volume of the core, $L_{2}$ is the length from inlet to the given position, $L_{t}$ is the total length of the core (Fig. 16).

The change in average water saturation expected from evaporation into gas, averaged over the region upstream of the given, is

$$
\Delta S_{\mathrm{w}}=N_{v 2} \times\left(4.18 \times 10^{-4}\right) .
$$

This estimate makes many assumptions. It averages the change in water saturation from evaporation upstream of a given position, whereas, as shown in Fig. 10, water saturation is lower near the inlet than further downstream. It ignores the effects of capillary pressure, drawing water back upstream to replace that evaporated. It is provided only to indicate that the expected effect of evaporation is a significant factor in the change in water saturations seen in the CT measurements.

\section{References}

Alvarez, J.M., Rivas, H.J., Rossen, W.R.: Unified model for steady-state foam behavior at high and low foam qualities. SPE J 6(03), 325-333 (2001)

Boeije, C.S., Rossen, W.R.: Fitting foam-simulation-model parameters to data: I. co-injection of gas and liquid. SPE Reserv Eval Eng 18(02), 264-272 (2015)

Gong, J., Vincent-Bonnieu, S., Kamarul Bahrim, R.Z., et al.: Modelling of liquid injectivity in surfactantalternating-gas foam enhanced oil recovery. Accepted for publication by SPE Journal. SPE-190435-PA (2018)

Heller, J.P.: $\mathrm{CO}_{2}$ foams in enhanced oil recovery. In: Schramm, L.L. (ed.) Foams: fundamentals and applications in the petroleum industry, pp. 201-234. American Chemical Society, Washington, D.C. (1994)

Kibodeaux, K.R., Rossen, W.R.: Coreflood study of surfactant-alternating-gas foam processes: implications for field design. In: SPE westernregional meeting, Long Beach, California, USA (1997)

Kibodeaux, K.R., Zeilinger, S.C., Rossen, W.R.: Sensitivity study of foam diversion processes for matrix acidization. In: SPE annual technical conference and exhibition, New Orleans, Louisiana, USA (1994)

Kuehne, D.L., Ehman, D.I., Emanuel, A.S., Magnani, C.F.: Design and evaluation of a nitrogen-foam field trial. J Petrol Technol 42(02), 504-512 (1990)

Lake, L.W., Johns, R.T., Rossen, W.R., Pope, G.A.: Fundamentals of enhanced oil recovery. Society of Petroleum Engineers, Richardson (2014) 
Matthews, C.S.: Carbon dioxide flooding. In: Donaldson, E.C., Chilingarian, G.V., Yen, T.F. (eds.) Developments in petroleum science, pp. 129-156. Elsevier, Amsterdam (1989)

Martinsen, H.A., Vassenden, F.: Foam-assisted water alternating gas (FAWAG) process on snorre. In: European IOR symposium, Brighton, UK (1999)

Nguyen, Q.P., Currie, P.K., Zitha, P.L.J.: Determination of foam induced fluid partitioning in porous media using X-ray computed tomography. In: International symposium on oilfield chemistry, Houston, Texas, USA (2003)

Nguyen, Q.P., Zitha, P.L.J., Currie, P.K., Rossen, W.R.: CT study of liquid diversion with foam. SPE Prod. Oper. 24(01), 12-21 (2009)

Noh, M.H., Lake, L.W., Bryant, S.L., Araque-Martinez, A.N.: Implications of coupling fractional flow and geochemistry for $\mathrm{CO}_{2}$ injection in aquifers. SPE Reserv Eval Eng 10(04), 406-414 (2007)

O'Sullivan, T.D., Smith, N.: The solubility and partial molar volume of nitrogen and methane in water and in aqueous sodium chloride from 50 to $125^{\circ}$ and 100 to 600 atm. J. Phys. Chem. 74(7), 1460-1666 (1970)

Persoff, P., Radke, C.J., Pruess, K., Benson, S.M., Witherspoon, P.A.: A laboratory investigation of foam flow in sandstone at elevated pressure. Reserv Eng 6(03), 365-371 (1991)

Pickup, G.E., Jin, M., Mackay, E.J.: Simulation of near-well pressure build-up in models of $\mathrm{CO}_{2}$ injection. In: EAGE European conference on the mathematics of oil recovery, Biarritz, France (2012)

Rossen, W.R.: Foams in enhanced oil recovery. In: Prud'homme, R.K., Khan, S.A. (eds.) Foams: theory, measurements and applications, pp. 413-464. Marcel Dekker, New York City (1996)

Rossen, W.R., van Duijn, C.J., Nguyen, Q.P., Shen, C., Vikingstad, A.K.: Injection strategies to overcome gravity segregation in simultaneous gas and water injection into homogeneous reservoirs. SPE J 15(01), 76-90 (2010)

Schramm, L.L.: Foams: fundamentals and applications in the petroleum industry. American Chemical Society, Washington, D.C. (1994)

Shan, D., Rossen, W.R.: Optimal injection strategies for foam IOR. SPE J 9(02), 132-150 (2004)

Stone, H.L.: Vertical conformance in an alternating water miscible gas flood. In: SPE annual technical conference and exhibition, New Orleans, Louisiana, USA (1982)

Zeilinger, S.C., Wang, M., Kibodeaux, K.R., Rossen, W.R.: Improved prediction of foam diversion in matrix acidization. In: SPE production operations symposium, Oklahoma City, Oklahoma, USA (1995)

Publisher's Note Springer Nature remains neutral with regard to jurisdictional claims in published maps and institutional affiliations. 